\title{
Avaliação das mudanças cristalográficas e morfológicas com o aumento da temperatura de sinterização de pós de hidroxiapatita
}

\author{
AZEVEDO, A. G. S. ${ }^{1}$ \\ ${ }^{1}$ Químico graduado pela Universidade do Estado de Minas Gerais - UEMG. Mestrando em Física e \\ Química de Materiais pela Universidade Federal de São João del-Rei - UFSJ. \\ Autor correspondente: Adriano Galvão de Souza Azevedo - Rua Vereador Elí Araújo, 321 - Fábricas - \\ São João del-Rei -MG. E-mail: adrianogalvao26@yahoo.com.br
}

Recebido em: 30/10/2012 - Aprovado em: 23/12/2012 - Disponibilizado em: 30/12/2012

\begin{abstract}
Resumo
A utilização de materiais a base de fosfato de cálcio para a substituição de partes do osso humano removido por acidentes ou enfermidades tem sido objeto de pesquisas dentro das ciências dos materiais. A hidroxiapatita de cálcio se demonstrou bastante viável como material de fabricação de próteses e de enxertos ósseos, não apresentando rejeição do corpo humano e promovendo a osteoindução tecido natural/prótese. $\mathrm{O}$ entendimento dos mecanismos de obtenção desse material é de suma importância para se produzir materiais de elevada pureza e resistência mecânica. Vários parâmetros devem ser cuidadosamente controlados durante a síntese da hidroxiapatita visando à obtenção de um material com a microestrutura ideal para se promover a osteoindução após sua utilização. Os tratamentos térmicos envolvidos durante a síntese são fundamentais para se formar cristais bem ordenados e com maior resistência mecânica. As variações microestruturais sofridas pelos pós de hidroxiapatita em cinco diferentes temperaturas de tratamentos térmicos $\left(105,900,1000,1100\right.$ e $\left.1200{ }^{\circ} \mathrm{C}\right)$ foram avaliadas com intuito de se estabelecer a temperatura ideal de sinterização da matriz cerâmica. A temperatura de sinterização ideal foi a que conseguiu promover o aparecimento de características fundamentais para a posterior utilização do material como prótese ou enxerto ósseo.
\end{abstract}

Palavras-chave: hidroxiapatita, Sinterização, osteoindução

\begin{abstract}
The use of the base material of calcium phosphate for the substitution of parts of human bone removed by accidents or diseases has been the subject of research in materials science. The calcium hydroxyapatite been shown quite feasible as a material for manufacturing prosthesis and bone grafts, showing no rejection of the human body and promote the natural tissue osteoinduction / prosthesis. Understanding the mechanisms of obtaining such material is of paramount importance to produce materials of high purity and mechanical strength. Several parameters must be carefully controlled during synthesis of hydroxyapatite in order to obtain a material with the microstructure ideial to promote osteogenesis after use. The heat treatments involved during synthesis are fundamental to form well-ordered crystal and greater mechanical strength. Microstructural variations suffered by post hydroxyapatite at five different temperatures of thermal treatment $\left(105,900,1000,1100\right.$ and $\left.1200^{\circ} \mathrm{C}\right)$ were evaluated in order to establish the ideal temperature of sintering of the ceramic matrix. The sintering temperature was ideal that could promote the emergence of fundamental characteristics for the subsequent use of the material as a bone graft or prosthesis.
\end{abstract}

Keywords: Hydroxyapatite, Sintering, osteoinduction 


\section{Introdução}

$\mathrm{O}$ aumento das pesquisas na área da medicina, trás um aumento na qualidade de vida de pessoas que necessitam de tratamentos mais eficazes ou soluções para problemas desconhecidos. Pesquisas voltadas para descobertas e ou melhoramentos de materiais usados nas diversas áreas médicas - materiais cirúrgicos, materiais para substituições de tecidos ósseos - aumentam cada dia mais devido às necessidades e o aumento do número de enfermidades. Doenças ou fraturas que necessitam da remoção de partes do corpo humano são comuns nos hospitais do Brasil, representando um grande trauma para o paciente que necessitará se adaptar a novas formas de vida, dependendo dos membros que são removidos (GOUVEIA et al., 2006) (VALENTE, 1999). Lesões onde partes do tecido ósseo são removidas são normalmente encontradas em acidentes. Os membros são geralmente retirados para se evitar complicações posteriores ou quando a recuperação dessas partes afetadas já não é possível. A busca por novos materiais que substituíam essas partes perdidas é motivos de novas pesquisas na área de materiais (REBELO, 2006). Os materiais criados a partir dessas pesquisas podem ser metálicos, poliméricos, compósitos ou cerâmicos. Todos eles devem apresentar características essenciais como: Elevada resistência mecânica, não apresentar toxicidade, elevada resistência à fadiga, não ser solúvel nos fluidos corpóreos e se possível ter boa compatibilidade com o sistema biológico onde será implantado (VALENTE, 1999) (PEREIRA et al., 1999).

Vários materiais possuem
características ideais para serem
utilizados na projeção de próteses
substituintes de tecidos ósseos, mas
somente materiais a base de fosfato de
cálcio conseguem se ligar de maneira
efetiva no osso natural e promover sua
regeneração (osteoindução) (VALENTE, 1999). A hidroxiapatita é um mineral da família dos fosfatos e possui fórmula química $\mathrm{Ca}_{10}\left(\mathrm{PO}_{4}\right)_{6}(\mathrm{OH})_{2}$, representando uma hidroxiapatita (HAp) estequiométrica quando a razão $\mathrm{Ca} / \mathrm{P}$ do produto obtido for 1,667 (MAVROPOULOS, 1999) (REBELO, 2006). É um mineral raramente encontrado na natureza, mas é um constituinte da fase óssea em concentrações consideráveis - esse mineral compõe aproximadamente $70 \%$ da fase mineral dos ossos e dentes apresentando boa biocompatibilidade com enxertos produzidos a base de 
fosfatos (MAVROPOULOS, 1999) (MEURICE et al., 2012). É um mineral que sofre substituições na sua rede cristalina por íons de espécies contaminantes (GOUVEIA et al., 2006). Concentrações elevadas desses contaminantes alteram propriedades do material - estrutura, solubilidade, decomposição do material em fases mais simples durante o tratamento térmico- necessitando de uma síntese livre de contaminantes para que esses problemas possam ser evitados. (SUGIYAMA et al., 1999). A utilização da HAp contaminada como substituinte ósseo pode comprometer todo o processo de osteoindução, necessitando posteriormente a realização de cirurgias de reparo das próteses instaladas, o que representa um aumento nos custos dos hospitais e um transtorno para o paciente (VALENTE, 1999). Várias rotas de obtenção da hidroxiapatita estão disponíveis na literatura, podendo se alterar a rota para a obtenção de cristais com tamanhos diferentes ou até razões $\mathrm{Ca} / \mathrm{P}$ diferentes $\mathrm{da}$ estequiométrica (VALENTE, 1999) (NAYAK, 2010) (MADHAVI et al., 2005) (MAVIS; CU, 2000) (GARCIA et al., 2005) (COSTA et al., 2009).

Os métodos de síntese mais utilizados são os de precipitação por via úmida, Sol gel, hidrotermal, fusão dos sais precursores em temperaturas elevadas (ZHANG, Y. et al., 2010). Desses métodos citados, a precipitação por via úmida das soluções contendo os íons precursores é de mais fácil aplicação e menor custo operacional, sendo muitas vezes o método mais viável para a produção de HAp de enxertos artificiais (CUNHA et al., 2004). Em todas as rotas descritas para reações de obtenção da hidroxiapatita um posterior tratamento térmico (sinterização) é necessário para que rearranjos estruturais, densificação do material, diminuição da área superficial específica, aumento da resistência mecânica e aumento da cristalinidade possam ocorrer (AMINZARE et al., 2012) (HENRIQUE et al., 2011). O estudo das alterações estruturais devido as diferentes rotas já foi descrito na literatura por alguns autores, mas as modificações ocorridas devido diferentes temperaturas de sinterização ainda são parâmetros que devem ser avaliados para esse tipo de material (GARCIA et al., 2005).

O seguinte trabalho teve como objetivo sintetizar uma hidroxiapatita estequiométrica e avaliar as modificações estruturais ocorridas devido ao aumento da temperatura de 
sinterização. Temperaturas de 105, 900, 1000,1100 e $1200{ }^{\circ} \mathrm{C}$ foram as temperaturas escolhidas para os tratamentos térmicos por 2 horas. Alterações na cristalinidade, espécies presentes na matriz cerâmica, morfologia dos grãos obtidos e estabilidade térmica do material foram avaliadas nas diferentes temperaturas de sinterização.

O estudos das modificações ocorridas nos pós de hidroxiapatita devido diferentes temperaturas de sinterização nos ajudarão a entender os mecanismos que ocorrem no material e qual a temperatura ideal para se obter um material que possua todas as características necessárias para que sua utilização seja a mais efetiva possível no tratamento de substituições ósseas.

\section{Metodologia e Resultados}

O método de precipitação dos pós a partir de soluções contendo os íons precursores foi a rota escolhida para síntese do material em estudo. Soluções contendo 1,0 e 0,6 mol.L-1 de $\mathrm{CaCl}_{2} \cdot 2 \mathrm{H}_{2} \mathrm{O} \quad$ e $\quad\left(\mathrm{NH}_{4}\right)_{2} \mathrm{HPO}_{4}$ respectivamente foram preparadas com água MILIQ para a retirada de outros íons indesejados durante a síntese. A síntese foi realizada dentro de uma capela, livre de contaminantes externos e com o gotejamento da solução de fosfato controlado em aproximadamente $5 \mathrm{~mL}$ por minuto(GARCIA et al., 2005). O Becker onde a reação ocorreu foi vedado com um filme plástico para evitar ao máximo a entrada de $\mathrm{CO}_{2}$ atmosférico no sistema e consequentemente favorecer a formação de carboxiapatita (GOUVEIA et al., 2006). A reação se deu no meio básico, onde a formação de cristais de hidroxiapatita é favorecida (SANTOS, M. H. et al., 2004) (GARCIA et al., 2005) (COSTA et al., 2009). O pH das soluções foi controlado em $11 \mathrm{com}$ a adição de hidróxido de amônio concentrado - $30-32 \%$ - e a solução foi mantida em agitação e temperatura constante $\left(60^{\circ} \mathrm{C}\right)$ durante todo o tempo de gotejamento do $\left(\mathrm{NH}_{4}\right)_{2} \mathrm{HPO}_{4}$ em $\mathrm{CaCl}_{2}$. Após a adição das soluções, o precipitado formado foi deixado em agitação por mais 2 horas. De acordo com (LI, B. et al., 2009) \& (CUNHA et $a l ., 2004)$ a equação que governa a reação de obtenção da hidroxiapatita é a seguinte:

$$
\begin{aligned}
& \left(\mathrm{NH}_{4}\right)_{2} \mathrm{HPO}_{4}+\mathrm{CaCl}_{2} \cdot 2 \mathrm{H}_{2} \mathrm{O}+\mathrm{NH}_{4} \mathrm{OH} \\
& \rightarrow \mathrm{Ca}_{10}\left(\mathrm{PO}_{4}\right)_{6}(\mathrm{OH})_{2}+\mathrm{NH}_{4} \mathrm{Cl}+\mathrm{H}_{2} \mathrm{O} \\
& \mathrm{O} \text { precipitado foi retirado do } \\
& \text { agitador magnético e mantido em } \\
& \text { descanso por } 24 \text { horas. O material } \\
& \text { decantado foi separado do restante }
\end{aligned}
$$


através de filtração com bomba a vácuo.

O material foi lavado incessantemente até a total retirada do subproduto formado $\left(\mathrm{NH}_{4} \mathrm{Cl}\right)$ que foi confirmado através de teste negativo com solução de nitrato de prata $\left(\mathrm{AgNO}_{3}\right)$. O precipitado livre de íons cloreto foi seco em aproximadamente $105{ }^{\circ} \mathrm{C}$ por 24 horas. O material obtido foi então desaglomerado em almofariz de ágata e pesado em balança analítica para obtermos o rendimento da reação de síntese. Os reagentes foram pesados e as soluções foram preparadas para a obtenção de $30 \mathrm{~g}$ de Hidroxiapatita (HAp) com uma razão final $\mathrm{Ca} / \mathrm{P}=1,67$ o que corresponde a uma hidroxiapatita estequiométrica $\left(\mathrm{Ca}_{10}\left(\mathrm{PO}_{4}\right)_{6}(\mathrm{OH})_{2}\right)$. A pesagem demonstrou que o rendimento da reação de síntese foi de $89,83 \%$, com a obtenção de 26,953g de HAp seca.

Os processos de sinterização foram realizados nos fornos da marca EDG F-3000 do laboratório de pesquisa em química de materiais da UFSJ. A sinterização foi realizada em cadinhos de alumina em diferentes temperaturas $\left(900,1000,1100\right.$ e $\left.1200^{\circ} \mathrm{C}\right)$ por 2 horas. As amostras sinterizadas foram novamente pesadas para a obtenção da porcentagem de massa perdida durante o tratamento térmico. Os pós foram desaglomerados em almofariz de ágata e caracterizados por difração de raios $x$ (DRX), espectroscopia no infravermelho (IV), microscopia eletrônica de varredura (MEV), análise termo gravimétrica (TGA) e análise termo diferencial (DTA). As caracterizações demonstraram que a alteração da temperatura de sinterização influencia - mesmo quando pequenas alterações são realizadas - na morfologia e cristalinidade dos pós de hidroxiapatita.

Os difratogramas de raios $\mathrm{x}$ foram obtidos no difratômetro da marca Shimadzu do laboratório de pesquisas de materiais do departamento de ciências naturais (DCNat) da Universidade Federal de São João delRei - UFSJ. Os difratogramas foram obtidos utilizando radiação $\mathrm{CuK} \alpha, \lambda=$ 1,542 , com varredura de $10-802 \theta$ e operando a $30 \mathrm{mV}$ e $30 \mathrm{~mA}$. Os difratogramas obtidos demonstram que a fase predominante é a do mineral hidroxiapatita. A comparação dos picos das amostras sintetizadas com os padrões presentes na literatura mostram com clareza a efetividade da síntese e a cristalinidade do material obtido (MADHAVI et al., 2005) (SANTOS, M. L. et al., 2005). Picos característicos do mineral podem ser observados em $26,0-31,8-32,26-32,96-39,92-$ 
$46,82-48,2-49,63-50,52-51,3-$ $52,12-53,272 \theta$. Esses e outros picos de menor intensidade comprovam que o mineral de interesse se apresenta como fase majoritária do pó obtido, como pode ser observado na figura 1 .

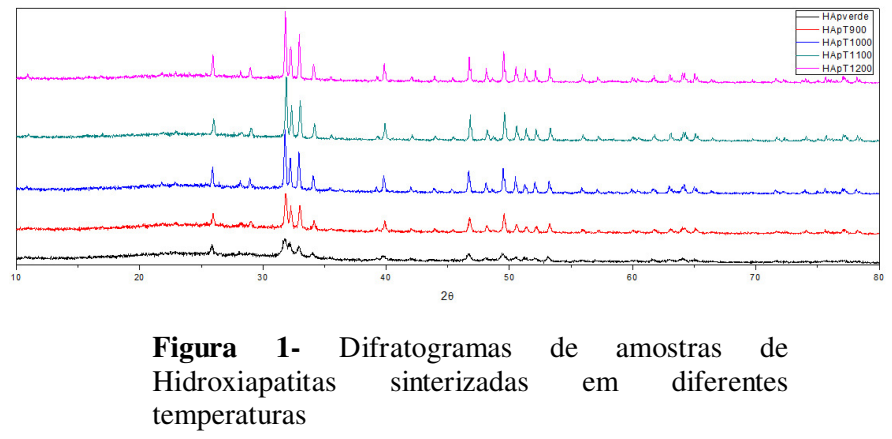

As intensidades dos picos demonstram que a cristalinidade do mineral apatita aumenta com o aumento da temperatura de sinterização. A formação de outras fases pode ocorrer devido ao aumento da temperatura, o que não foi observado no material sintetizado.

Na figura 2 temos o espectro de infravermelho obtido por um espectrômetro de infravermelho da marca Perkin Elmer. As amostras foram analisadas em pastilhas de $\mathrm{KBr}$.

A espectroscopia no infravermelho foi capaz de nos demonstrar a presença de espécies características da matriz cerâmica sintetizada. Não se observou nenhuma banda de vibração correspondente ao íon cloreto, demonstrando que o processo de lavagem foi satisfatório.

Figura 2 - Espectroscopia no Infravermelho de

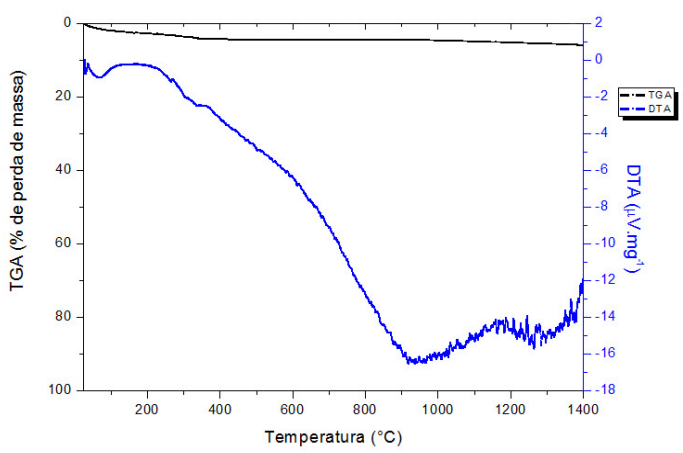

amostras de hidroxiapatitas sinterizadas a várias temperaturas

Os espectros no infravermelho demonstram que o aumento da temperatura de sinterização das amostram fazem com que as bandas correspondentes se alarguem. Outras bandas são mais bem observadas em temperaturas mais elevadas, como é o caso das bandas $570,0 \mathrm{~cm}^{-1}, 602,0 \mathrm{~cm}^{-1}$ e $3572 \mathrm{~cm}^{-1}$ (MADHAVI et al., 2005) (GOMES et al., 2007). Bandas correspondentes a espécies contaminantes, como carbonatos ou íons cloreto não foram observadas no espectro do infravermelho das amostras de hidroxiapatita, confirmando que o processo de síntese não permitiu a entrada de contaminantes (GOUVEIA et al., 2006). 


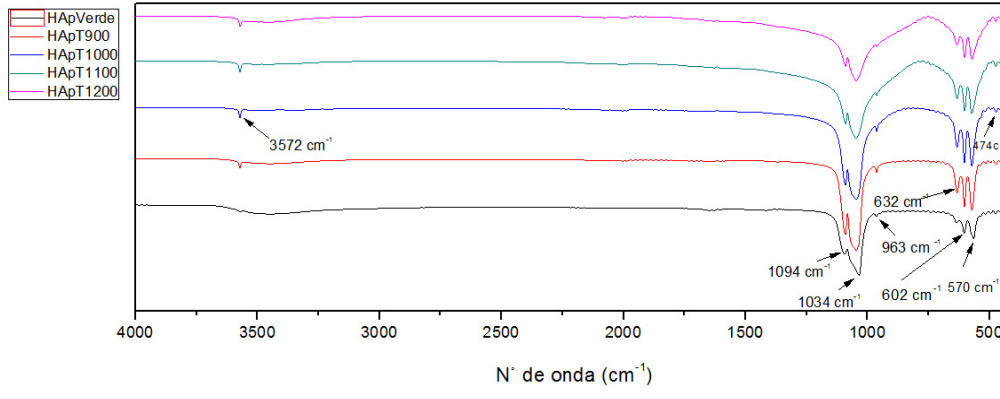

Figura 3 - Curvas TG-DTA do pó de HAp seco a $105^{\circ} \mathrm{C}$ por 24 horas.

As análises térmicas do material sintetizado foram obtidas utilizando um analisador térmico simultâneo TGA/DTA da marca Shimadzu do laboratório de análises térmicas da universidade federal de são João del-Rei. O material foi submetido a um aquecimento de $10{ }^{\circ} \mathrm{C}$ por minuto em uma atmosfera de $\mathrm{N}_{2}$ ultra puro da temperatura ambiente até $1400^{\circ} \mathrm{C}$. O material se demonstrou bastante estável dentro da temperatura de análise, onde a perda da massa total foi ínfima.

O material começa a ter uma perda de sua massa nos primeiros instantes da análise, o que pode estar associado à perda de moléculas de águas adsorvidas superficialmente. Nas análises de DTA das amostras, várias alterações podem ser observadas. Em aproximadamente $200^{\circ} \mathrm{C}$ tem-se uma curva de absorção de energia, o que pode ser associado à perda de água de hidratação. Em $250^{\circ} \mathrm{C}$ até $900^{\circ} \mathrm{C}$ temos uma curva muito acentuada de liberação de energia - reação exotérmica - que de acordo com a literatura, está associado a reações de rearranjo estrutural e reações oxidação de material orgânico remanescente (LACERDA et al., 2006). Não se observa maiores alterações de massa no material em temperaturas mais elevadas, demonstrando que a matriz cerâmica obtida é bastante estável. A perda final de massa do composto sinterizado está de acordo com as massas das amostras pesada antes e após os tratamentos térmicos por 2 horas em diferentes temperaturas, como pode ser observado na tabela 1.

Tabela 1 - Massa das amostras antes e após as sinterizações.

\begin{tabular}{l|l|l|l}
\hline $\begin{array}{l}\text { TEMPERATURA } \\
\text { DE }\end{array}$ & MASSA & MASSA & DE PERDA \\
SINTERIZAÇÃO & INICIAL & FINAL & MASSA \\
\hline $900^{\circ} \mathrm{C}$ & 3,028 & 2,885 & 4.722 \\
\hline $1000^{\circ} \mathrm{C}$ & 1,524 & 1,453 & 4.658 \\
\hline $1100^{\circ} \mathrm{C}$ & 1,501 & 1,44 & 4.063 \\
\hline $1200^{\circ} \mathrm{C}$ & 3,03 & 2,84 & 6.270 \\
\hline
\end{tabular}

A tabela 1 informa as massas iniciais de finais das amostras sinterizadas em diferentes temperaturas por 2 horas. Observa-se uma porcentagem de perda massa maior no processo de sinterização a $1200{ }^{\circ} \mathrm{C}$. 

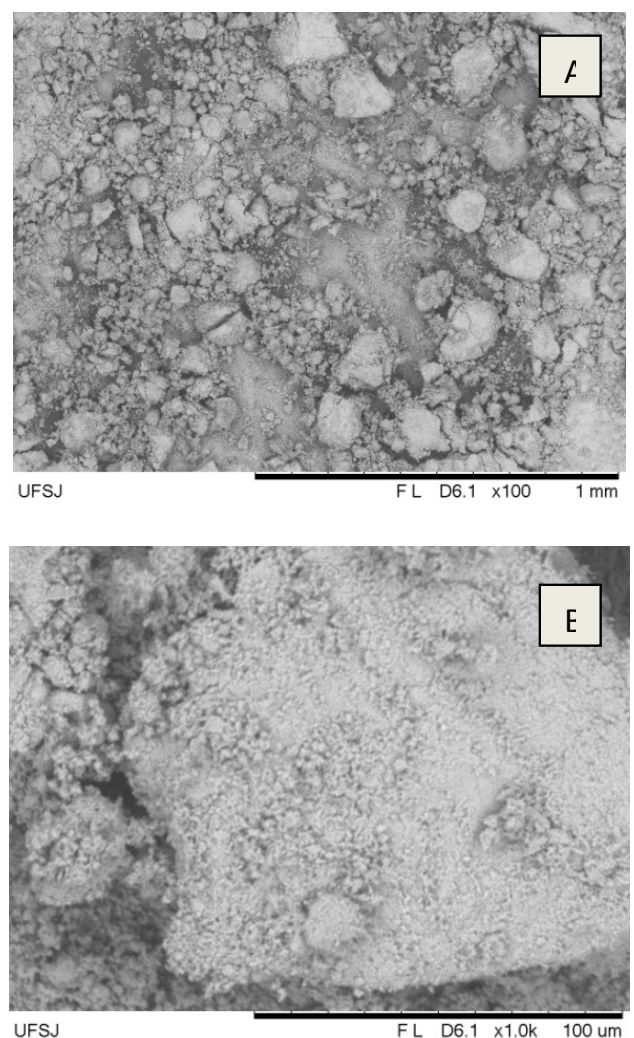

Figura 4 - MEV de amostras de HAp secas a $105^{\circ} \mathrm{C}$ por 24 horas. (a) aumento de 100X (b) aumento de $1000 \mathrm{X}$
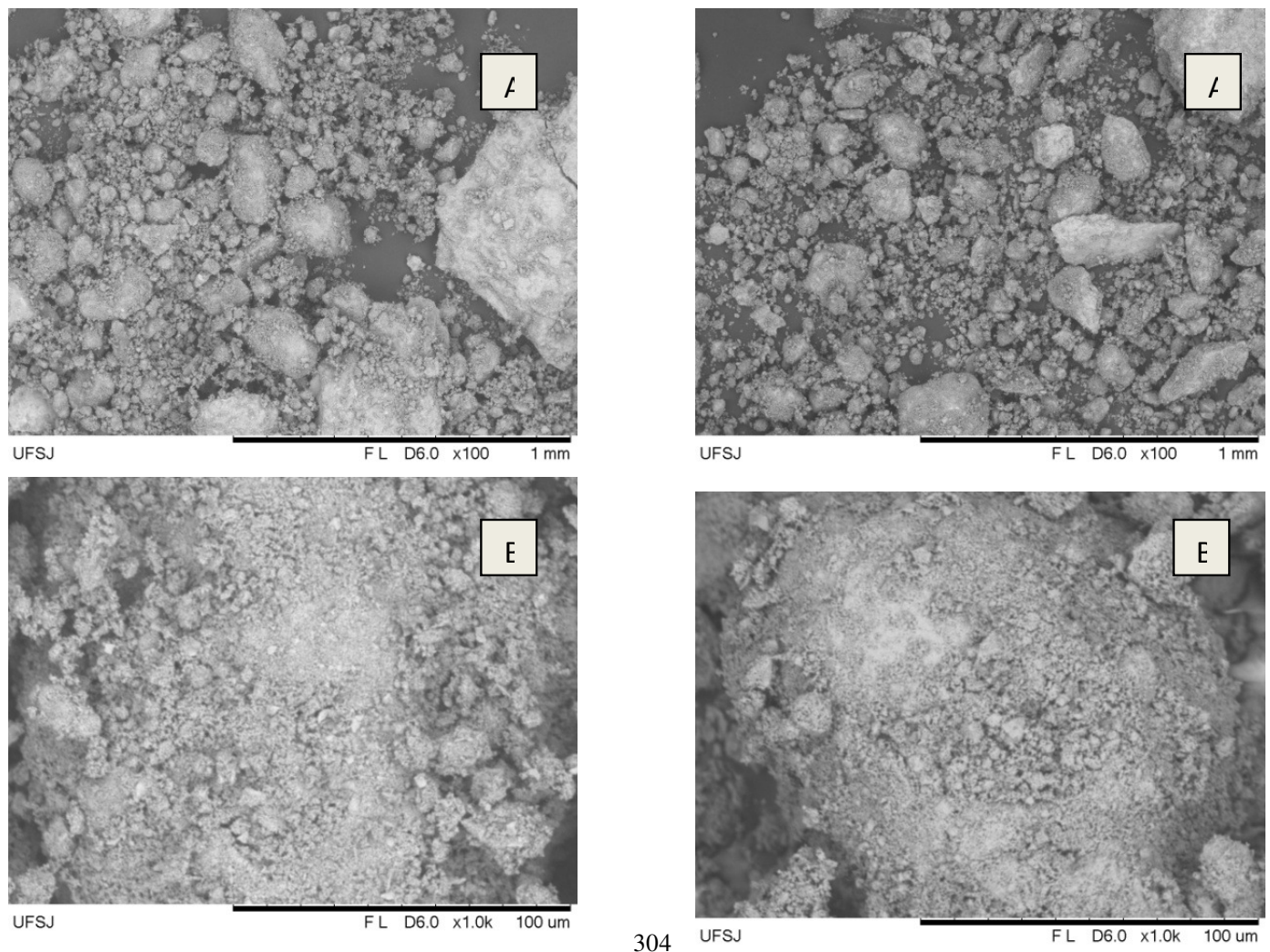

Kevista da Unıversıdade Vale do Kio verde, Três Cor . 

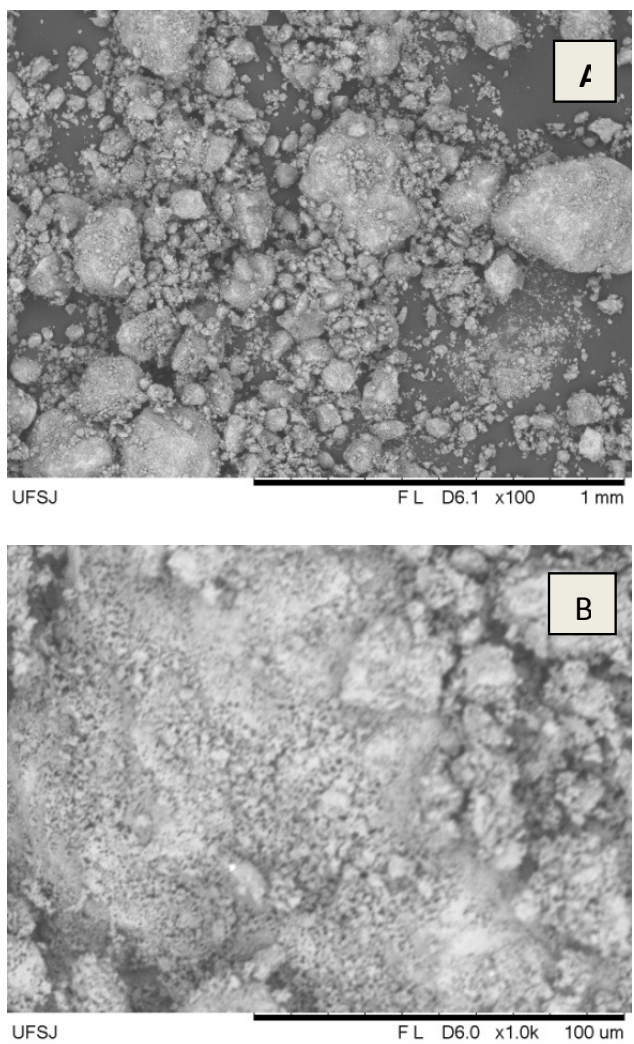

Figura 6 - MEV de amostras de HAp sinterizadas a $1200^{\circ} \mathrm{C}$ por 2 horas. (a) aumento de $100 \mathrm{X}$ (b) aumento de 1000X.

As micrografias eletrônicas de varredura das amostras foram realizadas em um aparelho Hitachi modelo TM3000 do laboratório de microscopia da Universidade Federal de São João del-Rei. Foram realizadas com aumento de 100X e 1000X onde se pode observar a morfologia das partículas obtidas pelo método de precipitação. Observa-se que a amostra que não passou pelo processo de sinterização (figura 4) apresenta morfologia diferenciada das outras amostras, apresentando maiores poros e partículas menos aglomeradas. Com o aumento da temperatura de sinterização das amostras as partículas vão se tornando mais densas, isso devido ao desaparecimento dos poros existentes (LACERDA et al., 2006). Na figura 8 já se observa uma hidroxiapatita muito densa, onde a presença de poros é quase inexistente, o que favorece o aumento da resistência mecânica da prótese fabricada com o material sinterizado nessa temperatura (WILLIAM D. CALLISTER, 2008).

\section{Conclusão}

As temperaturas de sinterização das matrizes cerâmicas de hidroxiapatita influenciaram de maneira considerável a microestrutura, como pode ser observado nos difratogramas obtidos e na morfologia dos grãos. A melhor temperatura de sinterização do material deve ser escolhida de acordo com os resultados obtidos nesse trabalho. Cada morfologia apresentará características microestruturais diferentes. Para a fabricação de uma prótese onde a resistência mecânica é uma característica desejável, aconselha-se utilizar uma HAp sinterizada em temperaturas mais elevadas. $\mathrm{O}$ aumento da temperatura de sinterização promove processos de difusão atômica dentro das partículas e consequentemente a diminuição dos tamanhos e números de poros. Essa diminuição eleva a resistência de materiais cerâmicos devido a maior dificuldade de propagação de trincas. Em temperaturas mais brandas, o material 
apresentou uma morfologia com muitos poros, o que favorece a osteointegração do material natural com o material sintético. $\mathrm{O}$ aumento do número de poros favorece a penetração de fluidos corpóreos no material, aumentando o número de ligações químicas entre proteínas presentes no organismo com a fase mineral artificial. Dessa maneira o osso natural tem o seu crescimento até a realização de uma interface osso natural e prótese, efetiva, o que corresponde a uma boa aceitação do material pelo sistema biológico.

\section{Referências Bibliográficas}

AMINZARE, M.; ESKANDARI, A.; BAROONIAN, M. H. et al. Hydroxyapatite nanocomposites: Synthesis, sintering and mechanical properties. Ceramics International, set 2012.

COSTA, A. C. F. M.; LIMA, M. G.; LIMA, L. H. M. A.; CORDEIRO, V. V.; VIANA, K. M. S. Hidroxiapatita : Obtenção, caracterização e aplicações. . [S.1: s.n.]. , 2009

CUNHA, S. M.; LAZAR, D. R. R.; USSUI, V.; LIMA, N. B.; BRESSIANI, A. H. A. SÍNTESE DE HIDROXIAPATITA POR PRECIPITAÇÃO HOMOGÊNEA. . Porto Alegre - RS: [s.n.]. , 2004

GARCIA, C.; CARLOS, P.; GAVIRIA, J. ESTUDIO DE ALGUNOS PARÁMETROS QUE DETERMINAN LA SÍNTESIS DE HIDROXIAPATITA POR LA RUTA DE PRECIPITACIÓN. . [S.l: s.n.]. , 2005
GOMES, J. F. P.; GRANADEIRO, C. C.; SILVA, M. A. et al. Optimização da reacção de síntese de hidroxiapatite por precipitação aquosa. Ciência e Tecnologia dos Materiais, v. 19, p. 7582, 2007.

GOUVEIA, D.; BRESSIANI, A.; BRESSIANI, J. EFEITO DO CARBONATO DURANTE A SÍNTESE DA HIDROXIAPATITA. metallum.com.br, n. 133, 2006.

HENRIQUE, B.; LOURENÇO, G.; SOUSA, E. D.; SILVA, G. CALCINAÇÃO NA SÍNTESE DE HIDROXIAPATITA. p. 1-4, 2011.

LACERDA, K.; LAMEIRAS, F.; SILVA, V. Síntese e caracterização de matrizes porosas de hidroxiapatita para aplicação como fontes radioativas em braquiterapia. Rem: Revista Escola de Minas, v. 59, n. 2, p. 217-223, 2006.

LI, B.; CHEN, X.; GUO, B. et al. Fabrication and cellular biocompatibility of porous carbonated biphasic calcium phosphate ceramics with a nanostructure. Acta

biomaterialia, v. 5 , n. 1, p. 134-43, jan 2009.

MADHAVI, S.; FERRARIS, C.;

WHITE, T. J. Synthesis and crystallization of macroporous hydroxyapatite. Journal of Solid State Chemistry, v. 178, n. 9, p. 2838-2845, set 2005 .

MAVIS, B.; CU, A. Dip Coating of Calcium Hydroxyapatite on Ti-6Al-4V Substrates. v. 91, p. 989-991, 2000.

MAVROPOULOS, E. A HIDROXIAPATITA COMO REMOVEDORA DE CHUMBO. [S.1.]: Fundação Oswaldo Cruz, 1999. 
MEURICE, E.; LERICHE, A.; HORNEZ, J.; BOUCHART, F. Functionalisation of porous hydroxyapatite for bone substitutes. v. 32, p. 2673-2678, 2012.

NAYAK, A. K. Hydroxyapatite Synthesis Methodologies : An Overview. v. 2, n. 2, p. 903-907, 2010.

PEREIRA, A.; VASCONCELOS, W.; ORÉFICE, R. Novos biomateriais: híbridos orgânico-Inorgânicos bioativos. Polim: Cienc e Tecnol, 1999.

REBELO, A. H. DOS S. Preparação de hidroxiapatite nanométrica com características adequadas ao processamento coloidal para aplicações biomédicas. [S.1.]: Universidade de Aveiro, 2006.

SANTOS, M. H.; OLIVEIRA, M. D.; PALHARES, L. et al. Synthesis Control and Characterization of Hydroxyapatite Prepared by Wet Precipitation Process. v. 7, n. 4, p. 625-630, 2004.

SANTOS, M. L.; FLORENTINO, A. O.; SAEKI, M. J. et al. Síntese de hidroxiapatita pelo método sol-gel utilizando precursores alternativos: nitrato de cálcio e ácido fosfórico. Eclética Química, v. 30, n. 3, p. 29-35, set 2005.

SUGIYAMA, S.; FUKUDA, N.;

MATSUMOTO, H. et al.

Interdependence of Anion and Cation Exchanges in Calcium Hydroxyapatite: $\mathrm{Pb}(2+)$ and $\mathrm{Cl}(-)$. Journal of colloid and interface science, v. 220, n. 2, p. 324-328, 15 dez 1999.

VALENTE, M. DE C. Síntese de hidroxiapatita e sua aplicação como biomaterial. [S.1.]: Universidade de São Paulo, 1999.
WILLIAM D. CALLISTER, J. Ciência e Engenharia de Materiais Uma Introduçao. [S.1: s.n.], 2008.

ZHANG, Y.; YOKOGAWA, Y.; FENG, X.; TAO, Y.; LI, Y. Preparation and properties of bimodal porous apatite ceramics through slip casting using different hydroxyapatite powders.

Ceramics International, v. 36, n. 1, p. 107-113, jan 2010. 